\title{
Réflexions sur l'évaluation des acquis cognitifs des étudiants au cycle supérieur au
}

Maroc.

Said Haouassia, Elmostapha Elkhouzai

Université Hassan $1^{\text {er }}$

Faculté des Sciences et Techniques

Département des Mathématiques et Informatique

Laboratoire de l'Ingénierie Didactique et Dynamique des Systèmes

(L.I.D.D.S)

26000, Settat, Maroc.

\section{INTRODUCTION}

\section{OBJECTIFS ET PLANS DE L'ARTICLE.}

L'objectif général du présent article est de relater un état des connaissances dont on dispose concernant l'évaluation des acquis cognitifs des étudiants au cycle supérieur au Maroc, pour proposer des pistes pour l'amélioration de la qualité des démarches d'apprentissage que connaissent les institutions supérieures de l'éducation et de la formation; notre article s'est articulé autour des quatre questions :de quelles informations nous disposons sur les acquis des étudiants ?quelle est la cause de la rareté des informations sur les acquis des étudiants ?Pourquoi devrait-on élargir notre savoir sur les acquis des étudiants ?Comment faire évoluer les pratiques d'évaluation des acquis ? L'exploration de cette question nous amène à reposer un certain nombre de questions quant au fonctionnement des formations universitaires, La méthodologie mise en œuvre a cherché de mettre en œuvre ces doubles objectifs, en diversifiant et corrélant les sources d'information. Nous avons privilégié Trois méthodes complémentaires. Premièrement, l'analyse de la situation actuelle, basée sur les quatre questions évoquées ci-dessus, s'est basée sur une étude de documents. Ainsi que Des entretiens semi-dirigés ont aussi été menés, en référence aux quatre questions, auprès d'un nombre restreint de responsables pédagogiques et d'étudiants de licence .Une troisième piste a procédé à une étude de cas, visant à comparer qualitativement et dans le détail, les pratiques d'évaluation des acquis des étudiants d'une même section dans deux universités différentes à enrichir l'ensemble du notre bilan sur l'évaluation des acquis des étudiants.

\section{QUE SAIT-ON DES ACQUIS DES ÉTUDIANTS ?}

À notre connaissance, aucune enquête n'avait pour objet d'identifier et à mesurer, à l'échelle de notre système éducatif, les acquisitions des étudiants aux différents au supérieur. De la même manière, aucun des programmes à caractère international de mesure des acquisitions, comme l'IEA ou PISA, n'a spécifiquement porté sur ce niveau des systèmes éducatifs. Il semble, que c'est la même tendance pour les enquêtes plus locale. Nous n'avons pas trouvé d' études au niveau national qui fournissent des indications sur les acquis des étudiants se regroupent autour de nos quatre thèmes : les compétences des étudiants à l'entrée 
des études, leur perception des compétences acquises à la fin de leurs études, leurs compétences méthodologiques et les rendements généraux de l'enseignement supérieur, par contre nous avons pu trouver à Echelle international des rares études qui fournissent quelques indications sur les acquis des étudiants se regroupent autour de nos quatre thèmes comme le cas de L'expérience belge MOHICAN . Des expériences de mesure locale, à l'échelle d'une institution ou d'une filière, sont également rapportées ponctuellement. Nous trouvons, Lebrun et Lega (1999) ont conçu un test portant sur la maîtrise de compétences scientifiques transversales (ex. savoir lire et interpréter un graphique). Ce test aide à dresser un portrait des pré acquis généraux des étudiants à l'entrée des filières scientifiques. Autres tests de même nature ont aussi été développés dans le domaine de la maîtrise de la langue maternelle (Defays et al., 2000).En évoquant ce cas nous pouvons signaler la coupure existant dans les pratiques d'évaluations dans le système éducatif national au niveau des cycles d'enseignement à savoir l'existence de tel pratiques de mesure des prérequis dans le cycle du collège et le lycée et son absence au supérieur. Pour la perception qu'ont les étudiants sur les compétences acquises , En aval de la formation, nous avons cherché des études qui ont également été consacrées à la perception qu'ont les étudiants des compétences qu'ils pensent en avoir développées au cours de leurs études supérieures, au niveau international, et national . pour le cas international on trouve Par exemple, Drew (1998) qui a mené, par entretiens de groupe puis par questionnaires à grande échelle, une étude sur les compétences que les étudiants estiment avoir acquises durant leurs études supérieures..

Il faut enfin signaler que nous avons mené un entretien semi dérigé, auprès d'un nombre restreint de responsables pédagogiques et d'étudiants pour dresser l'inventaire des compétences telles qu'elles sont attendues. Il ne s'agit évidemment, de recensements d'acquis, même perçus, mais plutôt de souhaits, d'attentes, voire de doléances. Cependant, ces entretiens apportent un éclairage intéressant à la discussion sur les finalités des formations universitaires : elles montrent notamment qu'il peut exister un décalage important entre les finalités académiques, souvent exprimées selon des logiques de contenus disciplinaires, et les attentes du monde économique et social. À titre d'exemple, les formations supérieures en mathématiques orientées vers les applications ne contient pas une description intéressante des qualités attendues d'un cadre recruté à la sortie de ces filières au contraire en terme d'évaluation elles mesurent les acquisitions des savoirs disciplinaires. Ce manqué de liste de compétences justifie nos entretiens.

\section{DES INVENTAIRES DE COMPÉTENCES MÉTHODOLOGIQUES}

Nous avons vérifié dans nos entretiens avec les responsables pédagogiques la liste implicite ou explicite des compétences méthodologiques pour la discipline mathématiques, et nous avons aboutit à :

L'essentiel des acquis semble être le fait des deux premières années d'études sur les trois que compte « la licence ». Les établissements qui assurent davantage d'acquis à leurs étudiants répondent aux caractéristiques suivantes : ils favorisent des interactions fréquentes entre les professeurs et les étudiants, leurs cursus sont 
flexibles et enfin leur personnel enseignant possède un niveau de qualification élevé. Au sein d'un même établissement, l'ampleur des acquis dépend de l'investissement de l'étudiant dans ses études et de certaines dimensions pédagogiques (la présence d'évaluations formatives, par exemple), mais pas d'autres : en particulier, les méthodes d'enseignement en petits groupes ne semblent pas systématiquement plus efficaces que l'enseignement magistral en grand groupe. Quant aux caractéristiques des enseignants qui assurent des acquis plus importants chez leurs étudiants, il est intéressant de constater qu'elles correspondent, dans une large mesure, aux critères que les étudiants retiennent quand il s'agit de décrire les enseignants qu'ils jugent « efficaces » : maîtrise de la matière, enthousiasme, clarté dans les explications, gestion efficace du temps, capacité de faire comprendre par des exemples et des analogies, langage clair et précis, capacité d'écoute des étudiants et de leurs problèmes.

Il est à noter que nous nous sommes intéressés aux savoirs cognitifs estimés à partir d'études de quelques modèles d'examens et de contrôles en mathématiques, et poser ce volet dans nos entretiens avec les responsables pédagogiques, il est intéressant d'observer est ce que les étudiants progressent aussi dans le domaine des savoir-faire cognitifs plus larges? Vue la rareté des informations concernant ce volet, surtout le manqué d'études et enquêtes national sur le sujet acquisition des savoirs cognitifs dans le sens dynamique c'est à dire à travers sa mesure à la fin d' un cycle ou tout le cursus de formation d'un étudiant, nous nous sommes bases sur la synthèse des travaux de Pascarella et Terenzini. Les principaux acquis répertoriés par la synthèse de Pascarella et Terenzini.

Une rapide comparaison montre que les acquis de savoir-faire cognitifs semblent d'une plus grande ampleur que les acquis de connaissances, ce qui rejoint d'une certaine manière le point de vue des étudiants . Par exemple, en se basant sur les résultats d'élèves aux tests de raisonnement abstrait à la sortie du secondaire, Pascarella et Terenzini estiment que 50\% des compétences de ce type, telles qu'on les observe chez les étudiants à la fin du « bachelor's degree », sont effectivement mises en place durant ces études. L'analyse des « effets nets » montre, de plus, que ces acquis sont principalement dus à l'enseignement supérieur lui-même et que l'essentiel des progrès enregistrés dans ces domaines lui est donc imputable. Les établissements les plus efficaces à développer des savoir-faire cognitifs généraux répondent aux caractéristiques suivantes : ils sont sélectifs, de petite taille et animés d'un " éthos institutionnel », comme l'appellent les auteurs, qui suscite un investissement de la part de l'étudiant. Les méthodes d'enseignement n'ont que peu d'influence sur ces acquis, à l'exception de l'ancrage de l'enseignement de ces savoir-faire au sein même des disciplines constitutives de la filière d'études.

Autrement dit, le raisonnement critique a d'autant plus de chance d'être maîtrisé par les étudiants qu'il leur aura été enseigné à propos de faits liés à leur domaine d'études.

Par contre pour le cas des établissements ouverts dans le système Marocain, du genre les facultés des sciences et sciences juridiques dont figurent les 
mathématiques comme discipline composante du cursus des étudiants en comparaissant aux facultés des sciences et techniques, nous trouvons que :

- $\quad$ les acquis des étudiants ne sont pas suffisamment explicités, identifiés et répertoriés ;

- $\quad$ les acquis majeurs, aux yeux des étudiants, ne sont peut-être pas ceux qui sont au cœur des examens traditionnels ;

- $\quad$ si l'université concentrait ses pratiques d'évaluation sur ces acquis essentiels, on peut faire l'hypothèse qu'elle parviendrait à les développer davantage.

\section{POURQUOI SAIT-ON SI PEU DE CHOSES SUR LES PRATIQUES D'ÉVALUATION DES ACQUIS À L'UNIVERSITE}

Les pratiques réelles d'évaluation des acquis des étudiants sont encore mal connues.

De même, on connaît mal la perception qu'ont les étudiants des pratiques d'évaluation. On sait quand même qu'une plus grande cohérence des pratiques d'évaluation et de notation constitue une de leurs principales revendications quand ils sont interrogés à propos des lacunes de l'enseignement universitaire pendant les entrevues que nous avons entamé avec les étudiants de notre échantillon. De même, quel que soit leur profil d'apprentissage, ils se montrent fort critiques sur les examens traditionnels. Ces derniers font, à leurs yeux, trop souvent appel à la seule mémorisation, pas assez à l'intégration des connaissances et les incitent à développer des apprentissages superficiels, qui ne leur seront d'aucune utilité dans leur vie professionnelle (Blais et al., 1997 ; LindBlom-Ylänne \& Lonka, 2001, S .haouassia 2016). Cependant, ils estiment aussi que les pratiques innovantes d'évaluation, comme les travaux de groupes évalués par les pairs, sont moins équitables que les examens traditionnels (Norton \& Brunas-Wagstaff, 2000,S.haouassia 2016). Par ailleurs, il nous a aussi été rapporté, au cours des entretiens, que les épreuves telles qu'elles sont organisées au sein des grands groupes du premier cycle ne semblent pas toujours avoir une grande crédibilité aux yeux des étudiants : ces derniers n'hésitent pas à mettre en doute la fiabilité des modes actuels d'évaluation des acquis.

Il existe cependant des études, mais en nombre limité, qui analysent minutieusement les pratiques d'évaluation des acquis des étudiants. Citons-en deux, parmi celles La premiière étude a été produite par l'Agence de modernisation des universités qui a consacré une de ses Rencontres à «L'organisation et la préparation des examens ». Le compte rendu de cette journée (Poirier, 2001) fournit d'intéressants renseignements sur les pratiques d'évaluation des acquis des étudiants. Il montre en particulier combien la lourdeur de l'organisation des examens, accentuée par la semestrialisation des enseignements, a eu pour conséquence fâcheuse de reléguer au second plan les réflexions pédagogiques sur l'évaluation.

Enfin, une d'étude à investiguer, dans le détail et de manière presque clinique, les comportements évaluatifs des enseignants du supérieur (York et al., 2000). Par la technique de la réflexion parlée ou par celle des entretiens de groupe, on cherche à savoir quels sont les comportements courants des enseignants du supérieur quand ils sont confrontés à des tâches authentiques d'évaluation. Par exemple, comment élaborent-ils leur jugement face à une copie ? Selon une démarche " platonicienne » de comparaison entre une copie modèle qu'ils ont en tête et les copies réelles ? Selon une approche intuitive, en regard de leur expérience antérieure de correcteur d'épreuves équivalentes ? En référence à une grille explicite de 
critères dont la pondération a été élaborée à l'avance ? S'appuient-ils sur un corrigé et si oui, quelle en est la nature ? Comment réagissent-ils face à un étudiant qui introduit dans sa réponse des éléments pertinents, mais qui n'étaient pas prévus dans leur corrigé ? ...

Quelles conclusions d'ensemble peut-on tirer de ces diverses études, ainsi que de notre recherche et réflexions?

\section{Conclusions}

La première conclusion qui se dégage, confirmée d'ailleurs par les entretiens, est que l'évaluation des acquis des étudiants est devenue une composante de plus en plus lourde du métier d'enseignant-chercheur. Les tâches d'évaluation occupent une place grandissante au sein de leurs activités., cette évolution semble aussi avoir produit des effets négatifs sur la qualité de l'évaluation : régression, voire disparition des oraux ; diminution des possibilités laissées aux étudiants de choisir leur formule d'examens ; non-tenues de certaines réunions de jury,soutiennent que la massification a entraîné, au Maroc, une diminution du contrôle continu et une modification de la nature même des épreuves, en particulier une régression des oraux, ce qui ne manque pas de modifier la nature des compétences développées . La multiplication d'examens déconnectés les uns des autres aboutit en effet au développement d'un " travail intellectuel morcelé et concentré sur les prochains « obstacles » au détriment de la réflexion et de l'approfondissement, attributs fondamentaux d'études universitaires

La deuxième conclusion majeure qui se dégage des études portant sur les pratiques d'évaluation dans le supérieur est la grande hétérogénéité de ces pratiques. la situation dans le supérieur contraste fortement avec les pratiques qui ont cours au premier et au second degré. À cause de la tradition d'autonomie des établissements d'enseignement universitaire et, nous ajouterions, de la tradition d'autonomie des personnes au sein de ces établissements l'évaluation des acquis, en ce compris la délivrance des diplômes, est de la compétence de chaque établissement et de chacun des enseignants, ces derniers évaluant leurs propres étudiants.

L'absence de standardisation des pratiques d'évaluation s'observe aussi à l'intérieur d'une même filière, même dans le cas de diplômes nationaux. Notre L'étude de cas, , a cherché à explorer les spécificités des pratiques d'évaluation de deux universités, pour une même filière, en ce qui concerne le contrôle des connaissances : chaque UFR organise librement, dans le cadre de son autonomie, la préparation des examens et décide des épreuves, ce qui pose la question de l'homogénéité des résultats . ce constat est Presque international nous orientons à voir L'étude de Jarousse et Michaux (2001) montre également que les modalités d'évaluation varient considérablement d'une filière à l'autre, mais aussi d'une université à l'autre pour une même filière. Après leur enquête auprès de 55 responsables de DEUG, les pratiques d'évaluation des acquis se différencient sur les points suivants.

la troisième conclusion majeure qui s'impose à la lecture des études sur les pratiques d'évaluation est que le système des examens universitaires fonctionne la plupart du temps en l'absence de définition, même locale, des connaissances et des compétences qui sont prévus en fin de formation.

Le premier facteur explicatif est que les formations elles-mêmes ne sont pas prioritairement conçues autour d'objectifs explicites d'acquisition de connaissances et de compétences. L'absence de données à propos des acquis, mise en évidence au point 2 , renvoie ici à la question de savoir quels acquis devraient être évalués et donc quels sont les objectifs assignés 
aux formations, Pour comprendre cet état de fait, il faudrait analyser plus longuement les modes de constitution des programmes de l'enseignement supérieur. De nombreux indices incitent à penser que la confection des programmes (le choix des matières, leur contenu, leur articulation et leur répartition horaire) est souvent plus « tributaire de la configuration des opportunités et des intérêts internes à l'institution " (Hutmacher, 2001, p.43) que d'un inventaire méthodique des compétences à développer, par exemple sur la base d'une analyse des conditions d'exercice des métiers auxquels donne accès le diplôme. Comme le signale cet auteur, il s'agit sans doute d'un des obstacles majeurs à une véritable professionnalisation des formations supérieures.

Pour améliorer la pratique d'évaluation À plus grande échelle, il pourrait enfin s'agir d'épreuves indicatives nationales, construites par un organisme indépendant sur la base, d'une part, des maquettes nationales et, d'autre part, de relevés des points communs qui apparaissent entre les objectifs annoncés par les différents établissements. On pourrait ici s'inspirer des méthodologies mises en œuvre dans les programmes internationaux d'évaluation des compétences des élèves de différents pays (par exemple, le récent programme PISA) ainsi que de celles qui ont été développées pour les autres niveaux scolaires. À la différence des opérations menées aux autres niveaux scolaires, il ne s'agirait pas d'évaluations de masse visant à mesurer, pour tel diplôme, les acquis de tous les étudiants, étant donné l'autonomie pédagogique des établissements et la diversité des programmes qu'elle entraine. Il ne s'agirait pas non plus de l'évaluation des établissements. Ces épreuves, menées sur des échantillons représentatifs d'étudiants, fourniraient un portrait indicatif des connaissances et des compétences appropriées par les étudiants de telle filière, à tel palier de leurs études. Elles seraient, bien entendu, distinctes des procédures de certification.

\section{Références.}

Blais J.-G., Laurier M., Van der Maren J.-M., Gervais C., Lévesque M., Pelletier G. (1997). L'évaluation des apprentissages à l'Université de Montréal et dans ses écoles affiliées. Montréal : Groupe de Recherche Interdisciplinaire en Pédagogie Universitaire.

Defays J.-M., Maréchal M. \& Melon S. (2000) (eds) La maîtrise du français : du niveau secondaire au niveau supérieur. Bruxelles : De Boeck.

Drew S. (1998). Students' perceptions of their learning outcomes. Teaching in Higher Education, 3 (2), 197-217.

Haouassia (2016) approche conceptuelle de l'évaluation des curriculums - cas des mathématiques-

Hutmacher W. (2001). L'université et les enjeux de la professionnalisation. Politiques d'éducation et de formation, 2 (2), 27-48.

LindBlom-Ylänne S. \& Lonka K. (2001). Students'perceptions of assessment practices in a traditional medical curriculum. Advances in health sciences education, 6, 121-140.

Norton L. \& Brunas-Wagstaff J. (2000). Students' perception of fairness of assessment. Paper given at ILTAC 2000 (The Institute for Learning and Teaching in higher education Annual Conference, College of Ripon \& York St-John, 27-29 june. 
Poirier Ch. (ed) (2001). L'organisation et la préparation des examens : Compte rendu des Rencontres de l'Agence de Modernisation des universités et des établissements. Paris : Agence de Modernisation des universités et des établissements

York M., Bridges P. \& Woolf H. (2000). Mark distributions and marking practices in UK higher education. Active learning in higher education, 1 (1), 7-27. 